\title{
Using Technology to Succeed in Theological Study
}

by Adam Lowe

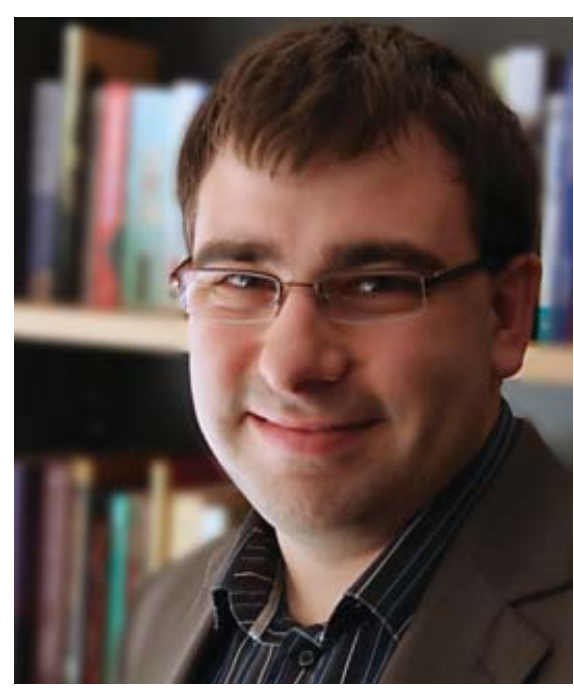

Using

Technology

to Succeed in

Theological

Study

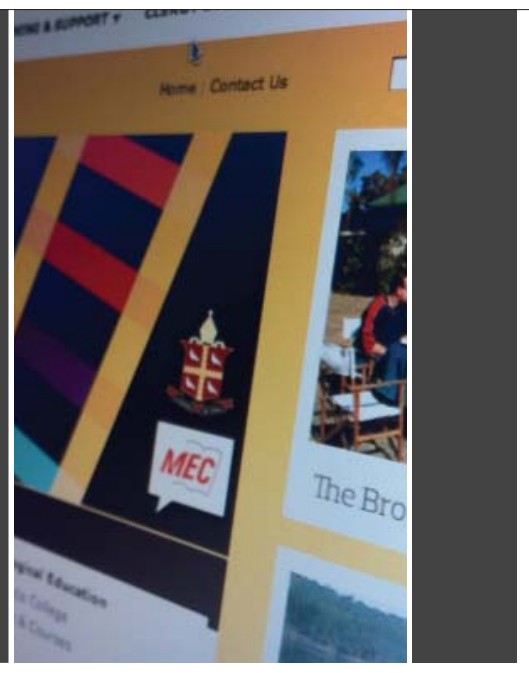

Adam Lowe

CSU, St Francis College (Brisbane) 


\section{Technology as a Tool}

[a] Backing Up \& Files

[b] Note-Taking

[c] Writing [d] Researching

[e] Using the Bible

\section{[f] Referencing}

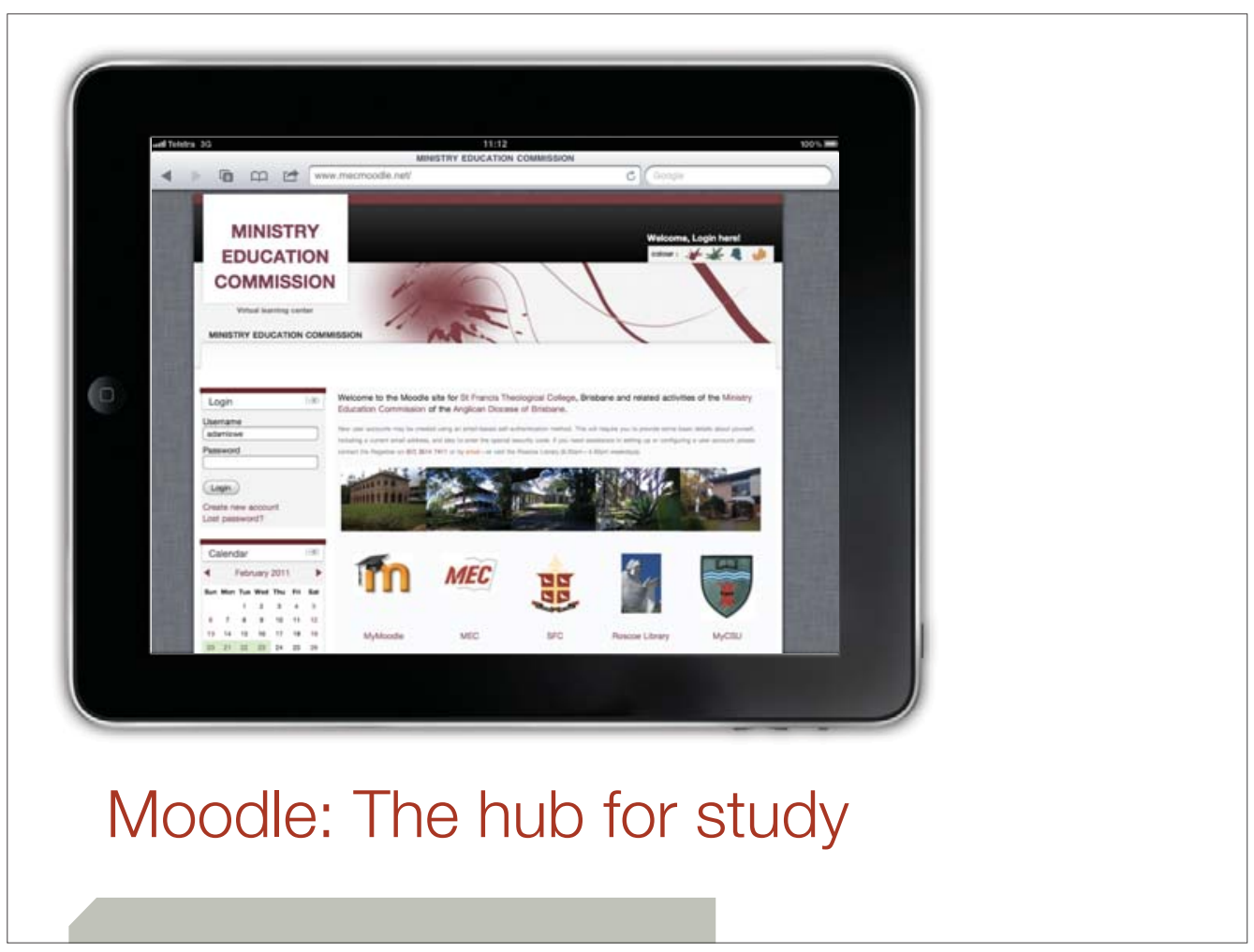




\section{Key Moodle Constructs}

- Homepage

- Course Page

- Overview \& Topics

- Participants

- Resources

- Files \& Folders

- LMS (Learning Management System)

- Text, Page, URL
- Activities

- Assignments

- Chat

- Choice (similar to a poll)

- Database

- Forum

- Glossary

- Lesson (a series of HTML Pages)

- Quiz

- SCORM

- Survey (Standardised)

- WIKI

- Workshop (with Peer Assessment)

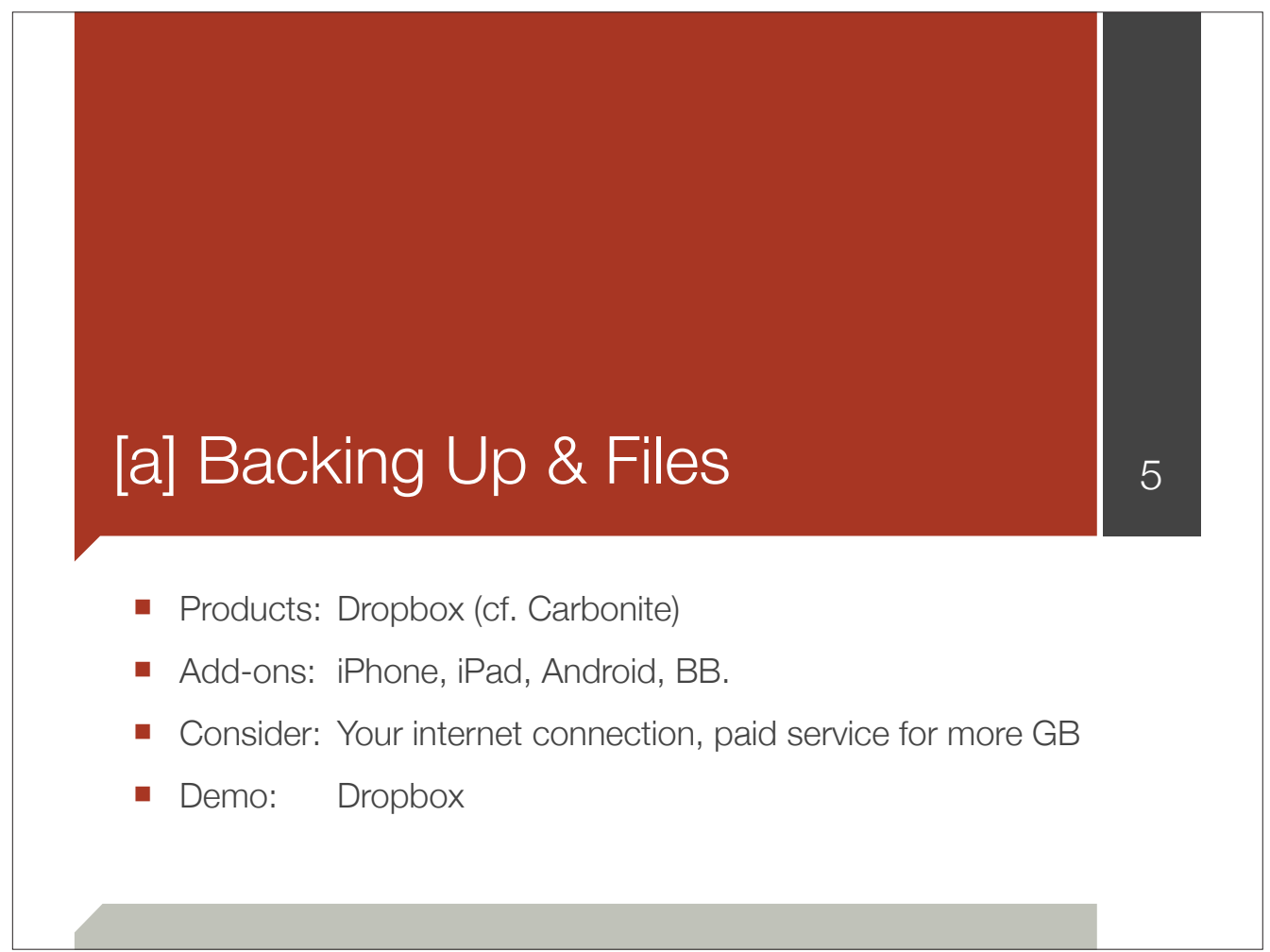



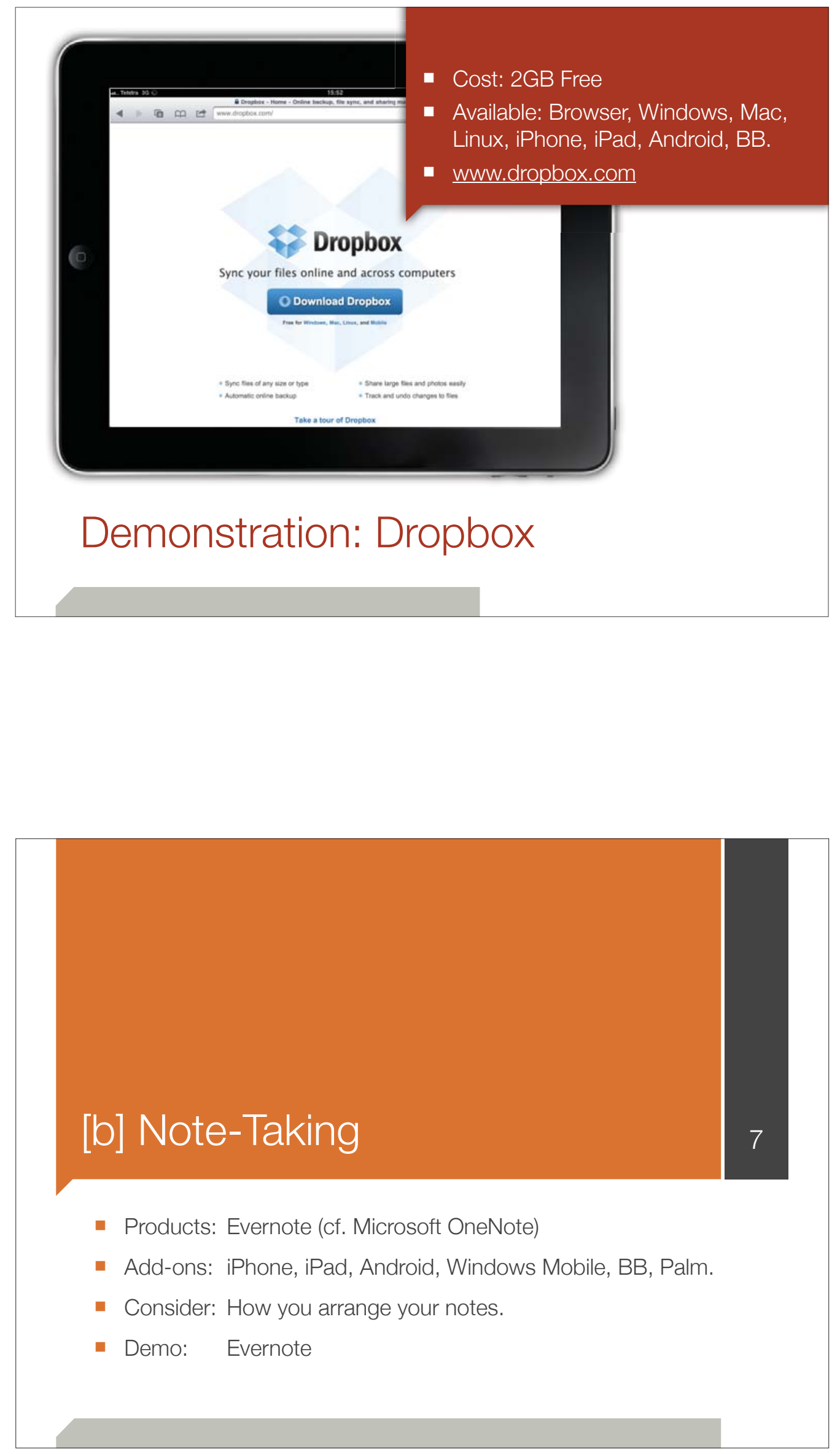

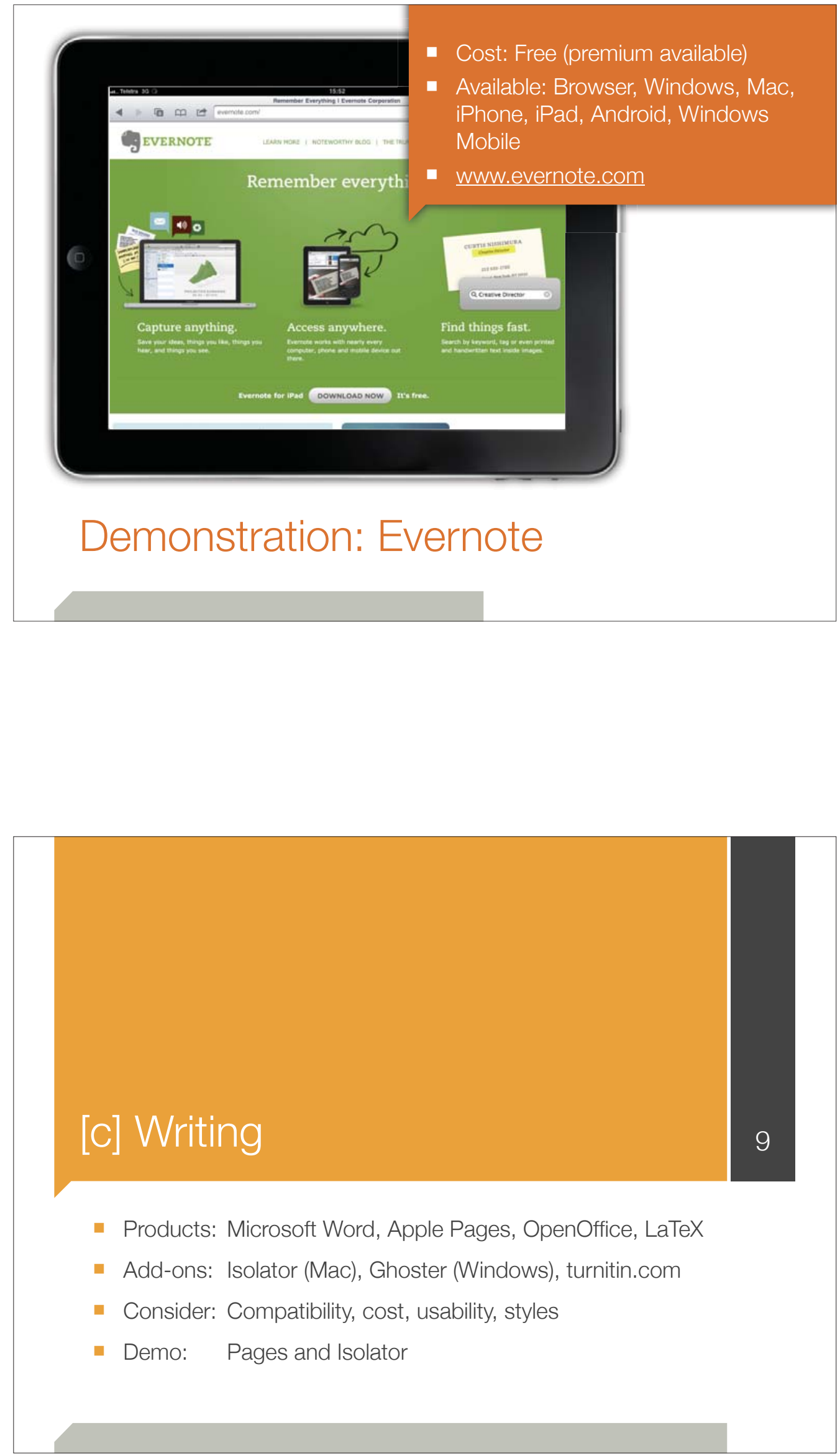

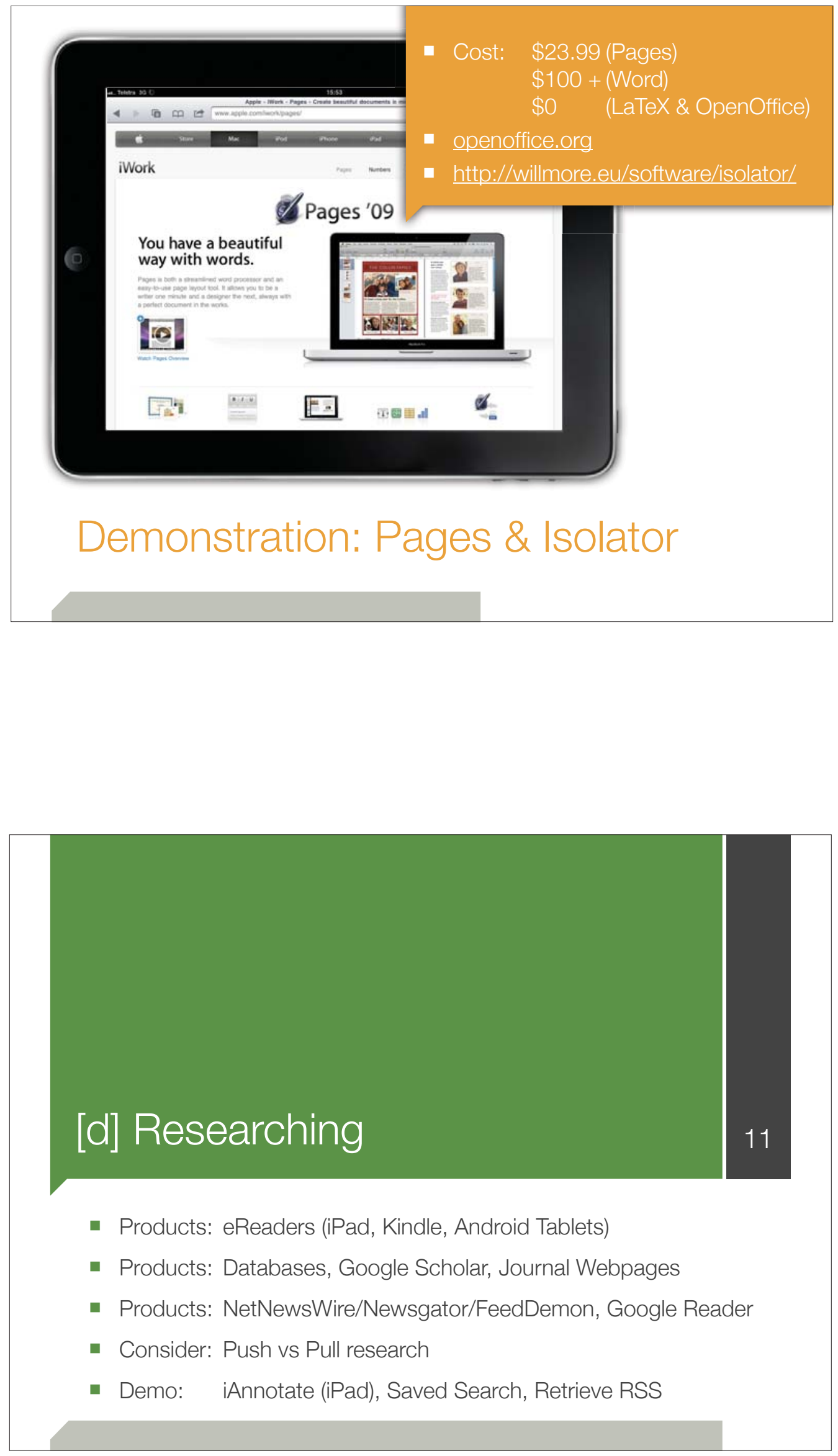

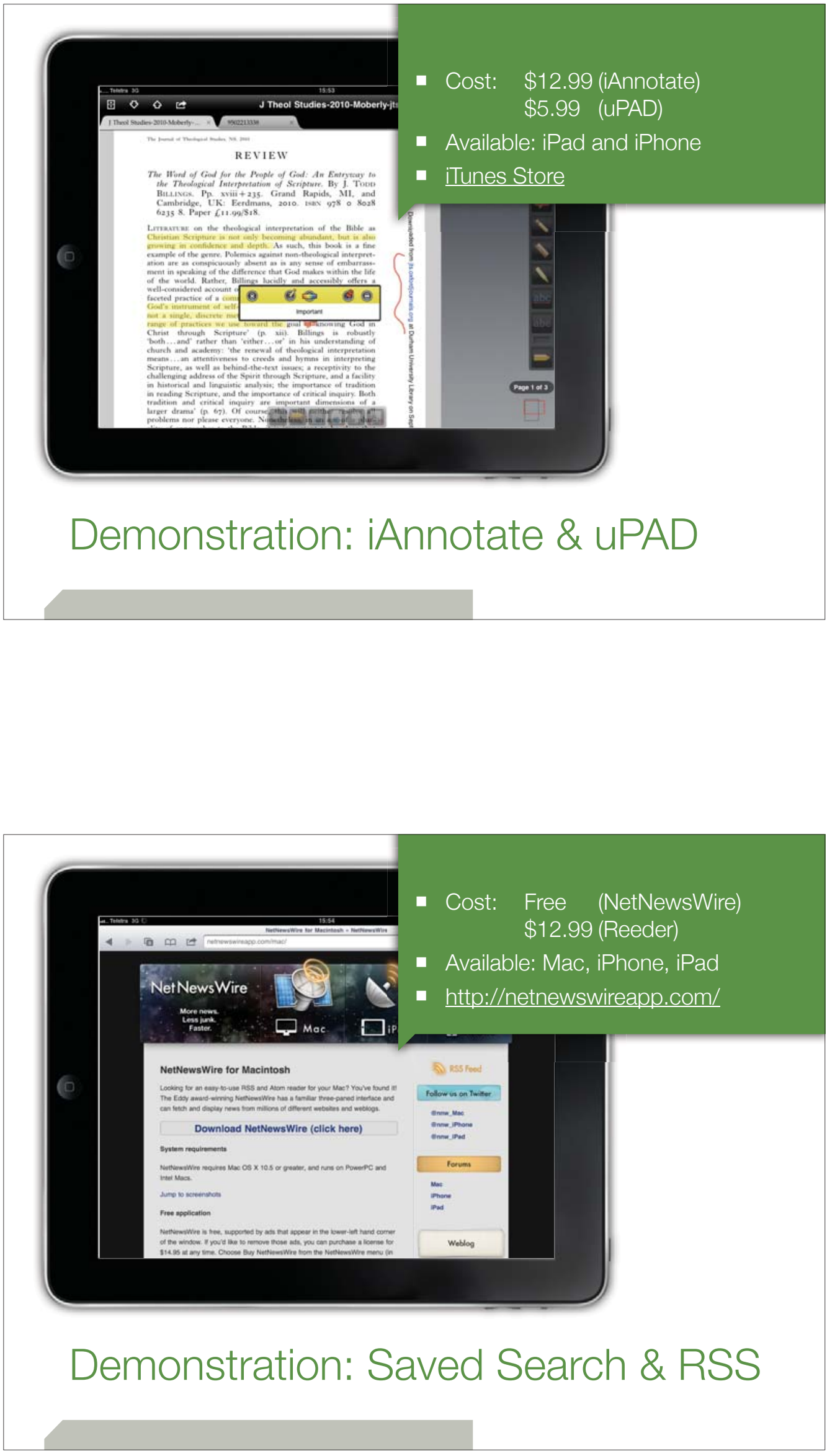
[e] Using the Bible

- Products: Accordance, BibleWorks, Logos, OliveTree

- Add-ons: iPhone, iPad, Android, Windows Mobile, BB, Palm.

- Consider: Usability, cost (over time)

- Demo: Accordance

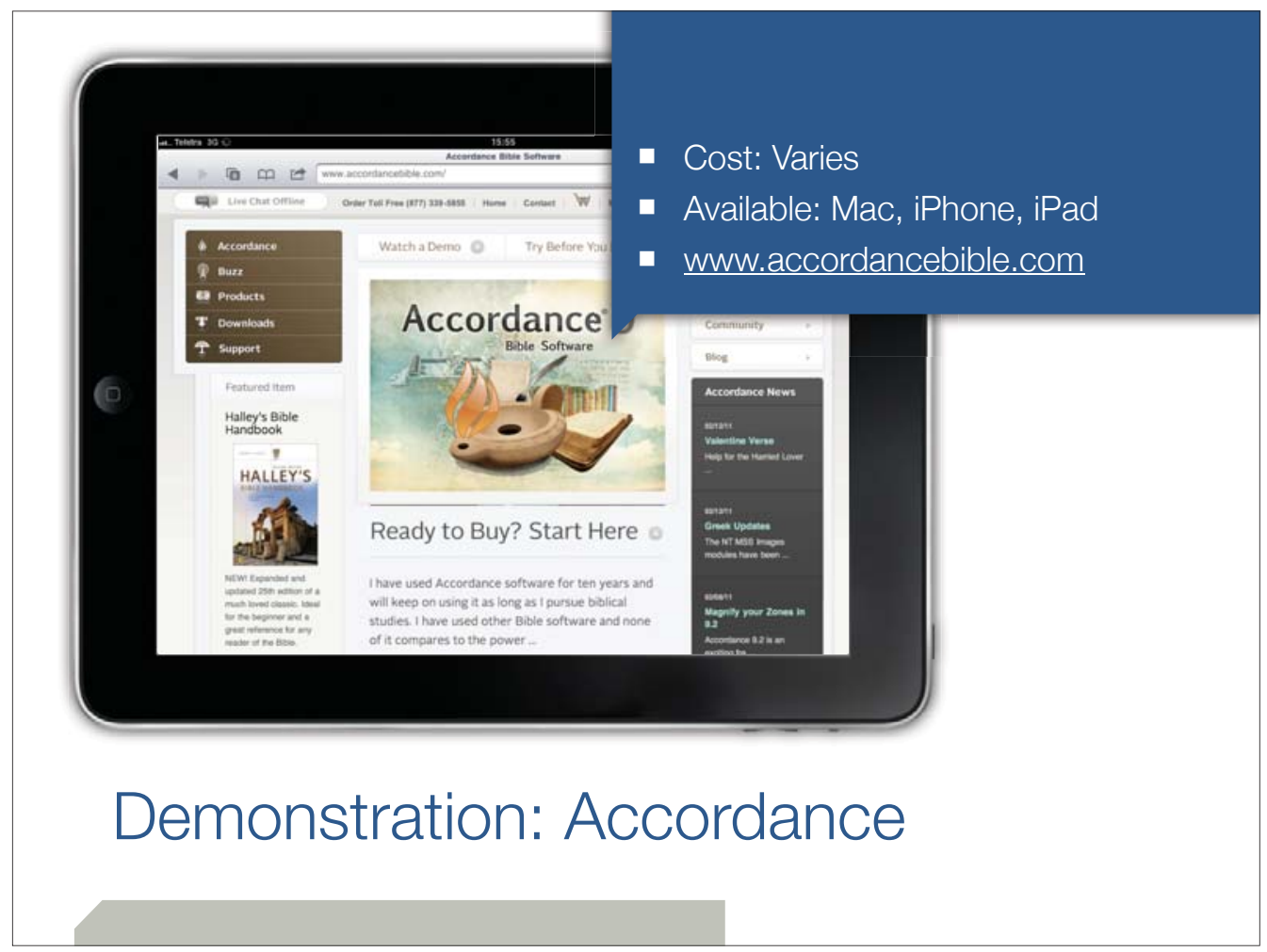




\section{[f] Referencing}

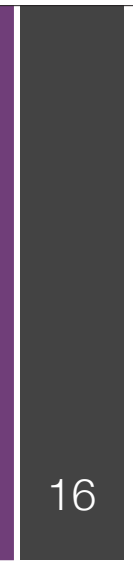

- Products: Endnote, BibTeX

- Consider: Building a reference library

- Demo: Endnote (organising your research, importing \& exporting references)

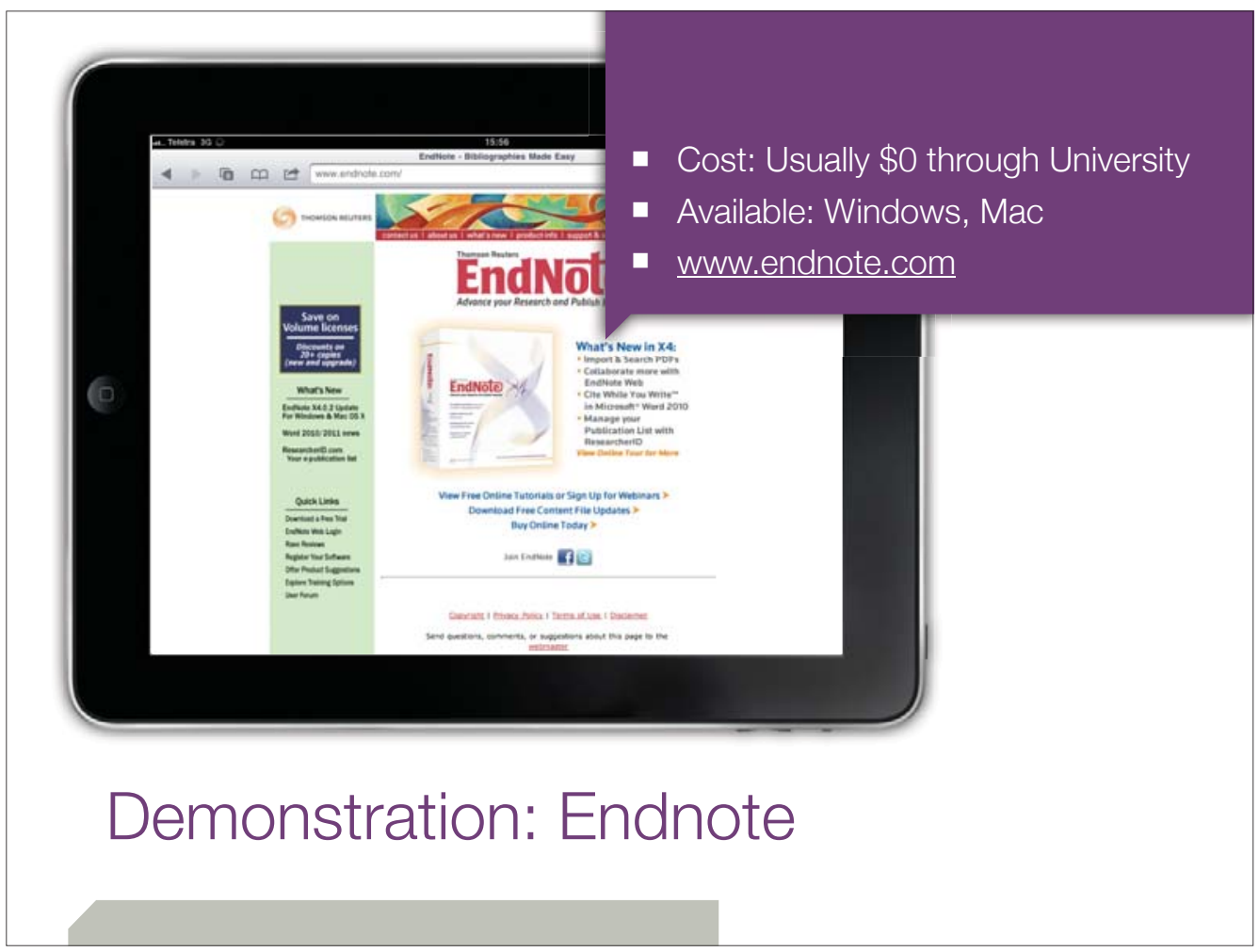




\begin{tabular}{|c|c|c|c|c|c|c|c|c|c|c|c|}
\hline & & & Windows & os $\mathrm{x}$ & Linux & iOs & Android & WMobile & Palm & BB & Browser \\
\hline \multicolumn{12}{|c|}{ [a] Backing Up \& Files } \\
\hline Dropbox & Free * & www.dropbox.com & 0 & 0 & 0 & 0 & 0 & & & 0 & O \\
\hline Carbonite & \$54.95/year & www.carbonite.com & 0 & 0 & & 0 & O & & & 0 & O \\
\hline \multicolumn{12}{|l|}{ [b] Note-Taking } \\
\hline Evernote & Free $^{*}$ & www.evernote.com & O & O & 0 & O & O & O & O & O & O \\
\hline OneNote & With Office & www.microsoft.com/onenote & O & & & 0 & & 0 & & & 0 \\
\hline \multicolumn{12}{|l|}{ [c] Writing } \\
\hline Pages & $\$ 23.99$ & Mac Applications Store & & 0 & & 0 & & & & & \\
\hline Microsoft Word & With Office & www.microsoft.com/office & O & O & & & & 0 & & & O \\
\hline OpenOffice & Free & www.openoffice.org & O & 0 & O & & & & & & \\
\hline LaTeX & Free & www.latex-project.org & 0 & O & O & & & & & & \\
\hline Isolator & Free & willmore.eu/software/isolator/ & & O & & & & & & & \\
\hline Ghoster & Free & Search donationcoder.com & O & & & & & & & & \\
\hline TurnltIn & Free & Through CSU Website & & & & & & & & & O \\
\hline \multicolumn{12}{|l|}{ [d] Researching } \\
\hline NetNewsWire & Free * $^{*}$ & www.netnewswireapp.com & & O & & O & & & & & $\bigcirc$ \\
\hline FeedDemon & Free & www.feeddemon.com & O & & & & & & & & \\
\hline GoogleReader & Free & www.google.com/reader & & & & & & & & & O \\
\hline InstaPaper & Free & www.instapaper.com & $\bigcirc$ & O & $\bigcirc$ & O & $\bigcirc$ & $\bigcirc$ & $\bigcirc$ & $\bigcirc$ & 0 \\
\hline UPAD & $\$ 5.99$ & iTunes Store & & & & 0 & & & & & \\
\hline iAnnotate & $\$ 12.99$ & iTunes Store & & & & 0 & & & & & \\
\hline \multicolumn{12}{|l|}{ [e] Using the Bible } \\
\hline Accordance & Varies & www.accordancebible.com & & 0 & & 0 & & & & & \\
\hline BibleWorks & Varies & www.bibleworks.com & 0 & & & & & & & & \\
\hline Logos & Varies & $\underline{w w w . l o g o s . c o m}$ & 0 & 0 & & 0 & 0 & & & & O \\
\hline OliveTree & Varies & www.olivetree.com & 0 & & & 0 & 0 & O & 0 & 0 & \\
\hline \multicolumn{12}{|l|}{ [f] Referencing } \\
\hline EndNote & Free & Through CSU/Uni Website & O & O & & & & & & & O \\
\hline BibTeX & Free & www.bibtex.org & 0 & 0 & 0 & & & & & & \\
\hline
\end{tabular}

